\title{
Defining pharmacology of natural products in the 21st century - challenge on multiple fronts
}

\section{Ding-Feng Su* \\ Department of Pharmacology, Second Military Medical University, Shanghai, China \\ ${ }^{*}$ Correspondence: dfsu2008@gmail.com}

Natural products are of significant interest to us in many ways. The air we breathe, the water we drink, the foods we eat are all natural products. Many drug products and toxins are also "natural".

Natural product sciences now face challenges at many fronts. At a global and economical level, biodiversity is diminishing everyday as the rainforest gives away to farmland and the coral reef is destroyed by pollution. As a result, many potentially valuable natural products are lost forever before we even know their very existence. An immediate implication beyond the direct loss is that we have less natural products to "copy" from. We pharmacologists may not have the power to change the world, but we can certainly contribute.

At a social level, natural products are generally perceived as safe and largely devoid of side effects. This belief has been taken advantage of to promote "natural health products". Beneficial effects of some are substantiated, but the only benefit of great many is only psychological. Some even created much damage to the believers. Aristolochic acid nephropathy is but only one example. Pharmacologists can, and are obliged to educate the public.

At a scientific level, pharmacologists who are interested in natural products also face many challenges.

First, rapid advances in related scientific disciplines (e.g. molecular biology, immunology) made us look like dinosaurs. We need to learn new concepts and technologies, and more importantly, to incorporate what we learn in our research.
Second, modern chemistry and material sciences have blurred the boundary between natural products and synthetic materials. A great many of substances are neither natural nor purely man-made. We need to venture out of our comfort zones to embrace such great opportunities.

Third, the use of natural products by human beings to combat diseases and promote health has never been with single known chemical identity until very recently. Many substances/recipes are known to have solid impact on human health; some have been validated by good clinical practice. Acceptance of application for herbal medications with multiple ingredients by the US FDA signals to the world a change of attitude towards acknowledging "good clinical observation”. This change probably also reflects a much more fundamental issue: the human body is a very complex organism. Very few diseases could be traced to a single cause. Multiple causes and pathological changes under most diseases call for intervention at multiple sites. One commonly encountered phenomenon in this area is the loss of biological activity in search of the "active compound". Some, and probably most, are due to technical reasons, but we need to consider the alternative: $1+1$ may be much more than 2. It is a general policy that studies accepted by this journal must be of known chemical entity or entities. This policy is needed to ensure the scientific quality but creates a set of problems. Frankly, we do not have a clear strategy to deal with this dilemma.
Last but not least, we also face challenges at a practical level. First, many natural products with biological activity have poor solubility in water. As a result, research on these natural products is often difficult. Second, natural products are often limited in supply. Semi or total synthesis is a discipline that attempts to address this issue. Third, many natural compounds with biological activity have complex chemical structures. Considering the almost infinite combination of chemical modifications, investigating the structure-activity relationship is tremendously time- and resource-consuming.

Great challenges are often synonymous to great opportunities. The creation of this journal is a testimony to our collective effort in rising up to the challenges and turn opportunities to advances. We welcome submission of scholarly manuscripts that may advance the pharmacology of natural sciences from every possible angle.

Received: 27 April 2010; accepted: 11 May 2010; published online: 28 May 2010.

Citation: Su DF (2010) Defining pharmacology of natural products in the 21st century - challenge on multiple fronts. Front. Pharmacol. 1:5. doi: 10.3389/ fphar.2010.00005

Thisarticlewassubmitted to FrontiersinEthnopharmacology, a specialty of Frontiers in Pharmacology.

Copyright $(\odot 2010 \mathrm{Su}$. This is an open-access article subject to an exclusive license agreement between the authors and the Frontiers Research Foundation, which permits unrestricted use, distribution, and reproduction in any medium, provided the original authors and source are credited. 